\title{
Deductive Nomological Model and Mathematics: Making Dissatisfaction more Satisfactory ${ }^{*}$
}

\author{
Daniele MOLININI
}

Received: 22.8.2012

Final Version: 10.1.2013

BIBLID [0495-4548 (2014) 29: 80; pp. 223-241]

DOI: $10.1387 /$ theoria. 6464

ABSTRACT: The discussion on mathematical explanation has inherited the same sense of dissatisfaction that philosophers of science expressed, in the context of scientific explanation, towards the deductive-nomological model. This model is regarded as unable to cover cases of bona fide mathematical explanations and, furthermore, it is largely ignored in the relevant literature. Surprisingly, the reasons for this ostracism are not sufficiently manifest. In this paper I explore a possible extension of the model to the case of mathematical explanations and I claim that there are at least two reasons to judge the deductive-nomological picture of explanation as inadequate in that context.

Keywords: Deductive-nomological model, Hempel, mathematical explanation, scientific explanation

RESUMEN: El debate sobre la explicación matemática ha heredado el mismo sentimiento de insatisfacción que los filósofos de la ciencia expresaron, en el contexto de la explicación científica, hacia el modelo nomológico deductivo. Este modelo se considera incapaz de cubrir casos de explicaciones matemáticas genuinas y, además, continúa siendo ignorado en gran medida en la literatura relevante. Sorprendentemente, las razones de este ostracismo no son suficientemente manifiestas. En este artículo exploro una posible extensión del modelo al caso de las explicaciones matemáticas y sostengo que hay por lo menos dos razones para juzgar la imagen nomológico deductiva de la explicación como inadecuada en este contexto.

Palabras clave: modelo nomológico deductivo, Hempel, explicacion matemática, explicación científica.

\section{Introduction: an Unsatisfactory Peacefulness}

The expression 'mathematical explanation' is generally used to indicate two distinct classes, or senses, of explanation: mathematical explanations in empirical sciences (MES) and mathematical explanations in mathematics (MEM). In both these explanations mathematics is regarded as playing an essential role in the explanation provided, i.e. mathematics unveils the reason why a particular state of affairs is true, although MES and MEM denote different things: the former are explanations in empirical sciences that make use of mathematics, whereas MEM refer to explanatory practices that take place within the

* An earlier version of this paper was presented at the CSHPS annual conference (Wilfrid Laurier University and University of Waterloo, May 2012.) and at the 4th conference of the SPS (University of Quebec in Montreal, June 2012). I'd like to thank the audience at those meetings for comments and feedback that have substantially improved this paper. I am also grateful to two anonymous referees for their valuable comments. This work was supported by a Fernand Braudel-IFER Outgoing Fellowship from the Fondation Maison des Sciences de l'Homme (FMSH, Paris) and the European Union (Action Marie Curie COFUND 7th PCRD). 
realm of mathematics itself. The existence of mathematical explanations, both in the sense of MES and of MEM, is now largely recognized in the literature (Mancosu 2011). There is no consensus, however, on how they work and whether they can be captured through a model. A possible strategy in the investigation of these explanations has been to assess a model of scientific explanation, such as Kitcher's unification model or Van Fraassen's pragmatic account, on a case of mathematical explanation (MES or MEM) which has been recognized as genuinely explanatory by the scientists in their practice. This is, for instance, the strategy chosen by Johannes Hafner and Paolo Mancosu in their studies on mathematical explanation (Hafner and Mancosu 2005, 2008). Similarly, David Sandborg tested Van Fraassen's model of explanation on a case recognized by the mathematician George Polya as a genuine example of MEM (Sandborg 1998). Besides providing philosophers with new insights into the mechanisms which would regulate MES and MEM, carrying out the analysis of mathematical explanation in this 'practice-driven' fashion also reveals the efficacy or the limitations of theories of scientific explanation and hence has been regarded as philosophically fruitful (Tappenden 2005).

Various traditional models of scientific explanation have been assessed in the context of mathematical explanation. It is therefore natural to ask what is the result of such an assessment in the case of the well-known deductive-nomological (D-N) model of scientific explanation. Now, whether we examine the contemporary studies on explanation and we turn our attention to the way in which the D-N model is discussed in connection with mathematical explanation, we find the following setting: the philosophical discussion about mathematical explanation has inherited the sense of dissatisfaction that philosophers of science expressed, in the context of scientific explanation, towards the $\mathrm{D}-\mathrm{N}$ model. With only the exception of a few papers, in fact, the -exponentially growing up- literature on mathematical explanation either rules out the possibility to use such a model for cases of MES and MEM, or simply ignores it, thus contributing to sanction its inefficacy. Even when the D-N model is mentioned in a paper on mathematical explanation, and therefore some attention is devoted to it, the author soon dismisses it as a good model to cover mathematical explanations. The consequence of this attitude is that any potential amendment to the model is cast aside from the beginning. Nevertheless, the reasons for this profound skepticism are not clear enough and they remain rather unexplored. As it was said above, other models of scientific explanation have been thought to extend to mathematical explanation and they have been subjected to an accurate analysis. These assessments have pointed to some problems of the models and therefore they disclosed the reasons why these models (or their extensions) are not good candidate to cover mathematical explanation in the classes MES and MEM. More importantly, they have led to progress in the philosophical analysis of scientific and mathematical explanation. On the other hand, there is no trace of such an evaluation for the case of the $\mathrm{D}-\mathrm{N}$ model in the context of mathematical explanation. Why is such a a classical theory of explanation in trouble when faced with a case of mathematical explanation? Surprisingly, although philosophers manifest a negative attitude towards the use of the model in the context of mathematical explanation, the literature has remained rather silent in this regard.

One reason for this philosophical peacefulness may be that there is an extraordinar- 
ily obvious reason for it $^{1}$. But what exactly is such an obvious reason? The D-N model was introduced as model of scientific explanation by Carl Hempel and Paul Oppenheim in 1948. As we are going to see in the next section, these authors conceived explanation as a mere logical deduction (the explanandum is deduced from laws of nature and initial conditions) whose constituents have to satisfy certain logical and empirical conditions of adequacy. On this account, it seems therefore meaningless to speak of 'mathematical explanation'. And this simply because according to this picture of explanation what is primary to explanation are laws of nature and initial (empirical) conditions, whereas mathematics has no explanatory import. Of course, this does not mean that Hempel and Oppenheim denied that mathematics contributes to explanation. Mathematics helps in formulating laws of nature, which are the very basic ingredient of the D-N model, and therefore it has an essential role in explaining scientific facts. However, the D-N model mirrors the idea that the explanatory power is not conveyed by the mathematics involved, thus ruling out the possibility of any genuine mathematical explanation. This would be the obvious reason why the $\mathrm{D}-\mathrm{N}$ model is banned from the debate on mathematical explanation. For instance, in comparing the main features of structural explanations (a particular instance of MES) with those which would fit a D-N explanation, Mauro Dorato and Laura Felline write:

However, as already implicitly shown in our former discussion, the D-N model cannot actually cover the presented case studies: when a phenomenon is explained structurally, the purely mathematical features of the model become essential, while laws of nature and initial conditions might be necessary but insufficient for the explanation (Dorato and Felline 2011, 172)

The point made here by Dorato and Felline, namely that the D-N model cannot cover mathematical explanations because these are essentially different from the kind of (scientific) explanations the D-N model was designed to cover within its structure, is perfectly reasonable and might well be adduced as the obvious reason why it is not worth using the D-N model in the context of mathematical explanation. Nevertheless, whether this is the reason for the ostracism towards the Hempelian account, it remains hidden in much of the debate on mathematical explanation. More generally, the inadequacy of the D-N model (i.e. inadequacy with respect to mathematical explanations in the forms MES and MEM) is implicit in a number of significant papers by some of the participants in the debate, e.g. in the works of Mark Colyvan and Christopher Pincock. Such inadequacy is indeed an implicit premise of their studies. But whether these authors consider that the D-N model is not able to capture the cases of mathematical explanation they focus on, they should at least offer (explicitly) the reason(s) why this is so. These authors do no offer, at least to my knowledge, these reasons. Their works suffer from this incompleteness, and the present paper is intended to contribute to the debate on mathematical explanation by providing explicit (and fresh) reasons that substantiate the inadequacy of the D-N model and complement Dorato and Felline's considerations. Besides, and more importantly for my purposes here, to observe that the $\mathrm{D}-\mathrm{N}$ model is not able to capture some cases of mathematical explanation does not exclude that it may

${ }^{1}$ Likewise in a sport magazine it would be superfluous to mention that the football team which won the last FIFA World Cup was not eliminated in the semi-finals. 
be modified and improved in order to capture genuine cases of MES or MEM. Indeed, whether a model of scientific explanation is dismissed as good candidate in the case of mathematical explanation, the reasons for this choice should be made clear in all their aspects. In such an evaluation, the possibility that a model could be successfully extended (where 'success' is given by the ability the model has to account for mathematical explanations) should be considered as well. This was the case for Kitcher's unification model or Van Fraassen's pragmatic approach, however this is not what happened for the D-N model. The dissatisfaction with respect to this model, or better towards such a picture of explanation, remains. But, again, this dissatisfaction has not been sufficiently settled.

The main purpose of this paper is to make this dissatisfaction more explicit and, consequently, more satisfactory. I will suggest that the D-N model cannot be extended, at least if we retain the original conception of explanation put forward by Hempel and Oppenheim, to cover cases of mathematical explanation. And this for two (related) difficulties that the D-N picture of explanation faces in the context of mathematical explanations in science and in mathematics: $(a)$ it cannot deal with mathematical operations or procedures which do not come under the form of statements but which are regarded as playing an explanatory role; $(b)$ it is not a sufficiently good indicator of the intuitions coming from scientific practice, thus imposing a picture of explanation which is not authentic (at least if we have faith in the intuitions of our scientists and mathematicians). In the following section I shall introduce the original model, together with three major criticisms that have been addressed to it. My aim in this section will be twofold. I shall provide the reader with a quick review of the account and, second, I shall show that the same dissatisfaction expressed towards the D-N model in the context of scientific explanation is present when the model is discussed in connection with mathematical explanations. Next, I shall consider a possible extension of the D-N account for mathematical explanations. My discussion will be based on a paper by Alan Baker (2005), in which such an idea is sketched (i.e. the idea that the D-N model can extend to mathematical explanations if properly modified). In section $4 \mathrm{I}$ shall point to the limitations that the extended version of the D-N model has in the context of mathematical explanation. The last section of the paper contains my conclusions.

\section{D-N model and Dissatisfaction}

In their famous essay Studies in the Logic of Explanation (1948), now considered one of the most influential studies in 20th century philosophy of science, Carl Hempel and Paul Oppenheim first advanced their D-N model of explanation (also known as covering law mode $)$. On this account, the explanation of a phenomenon is given in terms of a logical relation between a class of sentences and a singular sentence. A phenomenon is said to be explained when it is possible to deduce a sentence (the explanandum E), which describes the phenomenon to be explained, from some sentences (the explanans) which include initial conditions $C_{1}, \ldots, C_{k}$ and law-like generalizations $L_{1}, \ldots, L_{r}$. In order for the proposed explanation to be sound, its constituents have to satisfy certain conditions of adequacy, which may be divided into logical and empirical conditions (Hempel and Oppenheim 1948, 137): 
- Logical conditions of adequacy:

$R_{1}$ The explanandum must be a logical consequence of the explanans.

$R_{2}$ The explanans must contain general laws, and these must actually be required for the derivation of the explanandum and use no accidental generalizations ${ }^{2}$.

$R_{3}$ The explanans must have empirical content: that is, it must be capable, at least in principle, of test by experiment and observation.

- Empirical condition of adequacy:

$R_{4}$ The sentences constituting the explanans must be true.

As an illustration, consider we are asked why in April 1910 Halley's comet passed through its perihelion. We can derive a statement describing the position of the comet in April 1910 by using as premises Newton laws and some initial conditions (information about the masses of the Sun and the comet together with the present positions and velocities of each). This is a sound deductive argument in which Newtonian laws figure as essential premises, and therefore the D-N model considers it as an explanation of why in April 1910 Halley's comet came closest to the Sun.

Does the D-N model offer us the ultimate story about explanation in science? Unfortunately, it does not. Two classical counterexamples undermine the claim that the D-N model provides sufficient conditions for successful scientific explanation, while a third counterexamples attacks the claim that the model provides necessary conditions for explanation. I assume the reader is familiar with them, however I will shortly reconsider them here.

The first counterexample concerns the so called explanatory asymmetries (Bromberger 1966). Explanatory asymmetries appear when we have pairs of deductively valid arguments which rely on the same law(s) but which differ radically in explanatory potential. The classical example is that of the flagpole and the shadow. If we consider a flagpole and its shadow, from information about the height of the flagpole, the angle $\theta$ it makes with the Sun plus laws describing the rectilinear propagation of light, we can derive (a sentence about) the length of the shadow. This amounts to a reasonable explanation of the length of the shadow and fits Hempel's D-N model (the four conditions of adequacy $R_{1}, R_{2}, R_{3}$ and $R_{4}$ are satisfied). Nevertheless, equally, the deduction is perfectly legitimate, via the same laws and the same observation on the angle $\theta$, the other way around (we can derive the height of the tower from the length of the shadow). The problem with this second derivation, in the context of explanation, is that it seems nonsense to say that the length of the shadow explains the height of the flagpole. Indeed, it is difficult to regard this as a genuine explanation of $w h y$ the flagpole has that particular height. Although the former derivation corresponds to a reasonable explanation, the latter derivation intuitively fails to explain its conclusion. However, the D-N model considers as perfectly legitimate both directions of the explanation, thus lacking resources to discriminate the (intuitively)

\footnotetext{
${ }^{2}$ The term "laws" must be read as "laws of nature", as suggested by condition $R_{3}$.
} 
good explanation. A second counterexample to sufficiency is given by cases of explanatory irrelevances, i.e. situations where the logical derivation can satisfy the $\mathrm{D}-\mathrm{N}$ criteria but it should be considered a faulty explanation because it contains irrelevancies other than those associated with the directional features of explanation. The birth-control pills example given by Wesley Salmon exemplifies such situations (Salmon 1971, 34): $(L)$ All males who take birth control pills regularly fail to be pregnant; $(C)$ Mario Rossi is a male who has been taking birth control pills regularly; thus $(E)$ Mario Rossi fails to be pregnant. Despite the example fulfills the requirements of the D-N model, it would be manifestly bizarre to consider the sound deductive argument of premises $(L)$ and $(C)$ as a bona fide explanation of $(E)$.

The previous counterexamples show that if we want to preserve the D-N model we have to add to nomic expectability, i.e. "expectability on the basis of lawful connections" (Salmon 1989, 57), some other independent feature in order to account for directional features of explanation and ensure the explanatory relevance that is missing in the birthcontrol pills example. A third counterexample questions the claim that the D-N model describes necessary conditions for successful explanation. More precisely, it addresses Hempel's thesis of structural identity, according to which there exists a logical symmetry between explanation and prediction (Hempel and Oppenheim 1948, 139). The classical paresis and syphilis example, put forward by Michael Scriven (1962), is intended to show that this is not always the case. In short, the problem is that there exist explanations that have not a D-N structure: the condition cited as explaining $X$ fails to be nomologically sufficient for $X$ and it also fails to make $X$ high probable. The paresis-syphilis explanation considered by Scriven represents a case where an empirical phenomenon is explained but the D-N model is not able to exhibit its nomic expectability. In other words, nomic expectability is not a necessary condition for explanation.

After this short review of the D-N model, together with the main difficulties it faces, let me consider how the dissatisfaction which comes with the previous counterexamples is inherited when the D-N model is considered in the context of mathematical explanation. In order to discuss this point, however, I first need to highlight the very basic source of this dissatisfaction. To put it differently, I want to make clear why the previous counterexamples are regarded as legitimate to face the $\mathrm{D}-\mathrm{N}$ model and erode the Hempelian picture of explanation.

The three counterexamples above are implicitly based on the following intuition: what is taken to be genuinely explanatory should agree with what is taken to be explanatory in science, and more precisely with what is taken to be a bona fide explanation by the working scientists. The scientists' claims provide indicators of genuine explanation, and philosophers of science have to integrate their analysis in light of these claims. For instance, one deduction in the asymmetry problem is regarded as explanatory in science, whereas the other is not. In this case, the philosophical analysis of explanation has to turn to science and interpret philosophically the intuitions of the practicing scientist. A good account of explanation should be able to reflect these intuitions. And, in fact, this is the reason why the three examples above represent a problem for the D-N model. Of course, criticisms of the D-N model and statements about where and when it is explanatory, came from philosophers like Scriven and Bromberger. But the philosophical analysis put forward by these authors is sensitive to the claims of the practicing scientists 
and is supposed to mirror them. After all, we don't want our philosophical model to crash against the intuitions coming from scientific practice (or, even worse, against the intuitions coming from our best science). Observe that, contrary to what some philosophers engaged in the debate on mathematical explanations put forward, I am not suggesting here that claims from scientific practice should be considered as evidence of genuine explanation $^{3}$. Rather, I consider that these claims are indicators of the fact that there is a genuine mathematical explanation. An indicator needs some justification, whereas evidence does not. In other words, although we want our philosophical notion of mathematical explanation in science to reflect the scientists' intuitions, we do not want take these intuitions as genuine (unjustified) evidence for our philosophical work ${ }^{4}$.

Now, whether we consider mathematical explanations and we examine in what terms the D-N model is discussed in that context, we find the same sense of dissatisfaction that philosophers feel towards the model in the case of scientific explanation. When an empirical phenomenon or a mathematical fact is accounted for through mathematics, and the mathematical machinery is recognized by scientists as yielding explanatory power, philosophers of science want their model of explanation to recognize this case as a bona fide explanation. This would permit them to mirror the intuitions of scientists and account for their claims. However, it is simple to see why the D-N model fails to detect as explanatory such cases of mathematical explanation, thus betraying their expectations. According to condition $\mathrm{R}_{3}$, the explanans must have empirical content. Thus explanations with mathematical explanantia are automatically ruled out as genuine explanations by the D-N model. But, once again, to accept that would be quite unfavorable from a philosophical standpoint. Indeed, it would force us to deny that we are confronted with a genuine explanation and that mathematics plays an explanatory role - regardless whether the case is taken to be a genuine explanation by working scientists and mathematics is considered to play an explanatory role. This is the criticism that lies behind Dorato and Felline's observation (reported in the previous section). And, although in a different flavour, this is the criticism put forward by Robert Batterman in his book The Devil in the Details (2002). According to Batterman, the D-N model is not able to account for a particular kind of MES that he calls "asymptotic explanation" and whose existence is acknowledged by scientists in their practice ${ }^{5}$. In addition, Batterman notes that there is also a second argument against the possibility of using the D-N schema to cover cases of asymptotic explanations. His second observation concerns the use of idealization in the explanation involved. According to Hempel and Oppenheim, an adequate

${ }^{3}$ In the next section I will show how Alan Baker adopts this standpoint, i.e. he considers that scientists' judgements about explanatory value need to be taken at face value and provide such evidence.

${ }^{4}$ Similarly, Juha Saatsi observes that although philosophy of science should be sensitive to what scientists say about explanation, pronouncements and judgements from the practice of scientists do not provide any evidence for genuine explanation (Saatsi 2011, 153).

5 In footnote number 34 of his paper "Whose Devil? Which Details?” (2005), Gordon Belot suggests that there is no genuine fundamental distinction between the type of MES highlighted by Batterman, i.e. asymptotic explanation, and a classical D-N type explanation. In his reply to Belot, Batterman shows how asymptotic explanations cannot be given under the form of D-N derivations, and this because the nature of this class of MES is really different from that of the standard D-N type explanations (Batterman 2005). 
D-N explanation must meet the 'empirical condition of adequacy' $\left(\mathrm{R}_{4}\right)$, i.e. the sentences constituting the explanans must be true. Hence any explanation appealing to mathematical idealizations and idealized structures, which are false, is automatically excluded as good candidate for the D-N logical machinery (Batterman 2005, 162, footnote). Again, mathematical explanations are ruled out according to Hempel and Oppenheim's original conditions of adequacy.

In short, to accept the D-N model in the original form above simply means denying that mathematical explanations (MES and MEM) exist. Nevertheless, the intuitions from scientific practice seem to suggest that this is not true. And, after all, accounting for these intuitions through some conceptual framework is what philosophy of science is supposed to do, or at least one of its main tasks. There is, however, one question that we still want answered and that has not been addressed yet, namely: What about a possible implementation of the D-N model in the context of mathematical explanation? In the following section I shall turn to this issue.

\section{Extending the D-N model to Mathematical Explanations}

The final considerations of the previous section suggest that our acceptance of the D-N model and the existence of bona fide mathematical explanations are mutually exclusive. There remains, however, the possibility that the D-N account may be modified to account for mathematical explanations. In this section I am going to explore this option.

In his paper "Are there genuine mathematical explanations of physical phenomena?" (2005), Alan Baker presents a case of MES from evolutionary biology. The specific biological phenomenon concerns the life-cycle of an insect called periodical cicada. Why has this phenomenon caught the attention of philosophers of science? Its particularity lies in the fact that three species of cicada of the genus magicicada share the same unusual life-cycles, 13 or 17 (years), which are prime numbers. Although some features of the life-cycle could be explained by referring to ecological constraints (great duration of the cicada life-cycle and presence of two separate life-cycle durations within each cicada species in different regions) and evolutionary biological laws (the periodic-syncronized emergence of adult cicadas), the prime-numbered-year cicada life-cycle is quite mysterious and is in need of explanation. We want an answer to the following question: Why are the life-periods of the cicada prime?

Now, as Baker points out, two explanations based on the advantage of prime cycle periods have been offered: one based on avoiding predators, and the other on the avoidance of hybridization with other species. The former is based on the observation that the particular life-periods of cicadas avoid overlap with the life-periods of other periodical organisms and are therefore beneficial whether the other organisms are predators. The latter is based on the observation that these life-periods avoid overlap with different subspecies and are therefore beneficial since mating between subspecies would produce offspring that would not be coordinated with either subspecies. Both the explanations consider then that the particular life-periods 13 and 17 do minimize overlap with nearby (or lower) life-periods of other periodical organisms. Without entering here into the technicalities of the argument, Baker's observation is that such a minimum overlap is granted 
by a number theoretic result: 'prime periods minimize intersections compared to nonprime periods ${ }^{6}$. Both the explanations use this result as an essential element in giving the explanation for why is advantageous for the cicadas to have such a prime life-cycle. The number theoretic result is therefore essential to the structure of the general explanation, which makes also use of specific ecological facts and general biological laws, and answers the particular question 'Why are prime periods evolutionarily advantageous?'. The structure of the explanation is given by the following 5-step argument ${ }^{7}$ :

(1) Biological law: Having a life cycle period which minimizes intersection with other (nearby/ lower) periods is evolutionarily advantageous.

(2) Number theoretic theorem: prime periods minimize intersection (compared to non-prime periods)

From (1) and (2) we have:

(3) Mixed biological / mathematical law: Hence organisms with periodic life-cycles are likely to evolve periods that are prime.

Combining the previous result (3) with the following

(4) Ecological constraint: Cicadas in ecosystem type, E, are limited by biological constraints to periods from 14 to 18 years.

we obtain the prediction:

(5) Hence cicadas in ecosystem type, E, are likely to evolve 17 -year periods.

According to Baker, the fact that some biologists accept the explanation-schema above, which relies on the number theoretic result, provides evidence for the fact that the application of mathematics yields explanatory power ${ }^{8}$. However, to recognize that scientists consider this as a case of genuine mathematical explanation in science is not enough. It remains the difficult problem of determining, for the given explanation, where this explanatory power comes from, namely to account for the explanatory power of mathematics. This issue is raised by Baker in section 3 of his paper, titled "Is the cicada example a genuinely mathematical explanation?".

In the third part of his paper, Baker considers as possible candidates to cover his example of MES three accounts of scientific explanation: the causal model, the D-N model and Van Fraassen's pragmatic model. The causal account is soon rejected as a possible account for MES because of its incompatibility with any genuine mathematical

${ }^{6}$ The number theoretic result is actually a consequence of two lemmas (Baker 2005, 232).

${ }^{7}$ The same argument holds for cicadas having a life-cycle period of 13 years. As may be expected, in this case the ecological constraint (4) will be different.

${ }^{8}$ This claim is made more explicit in Baker (2009): "The way biologists talk and write about the cicada case suggests that they do take the mathematics to be explanatory, and this provides good grounds, at least prima facie, for adopting this same point of view" (Baker 2009, 625). 
explanation ${ }^{9}$. On the other hand, Baker claims that the D-N account and the pragmatic account "both support the claim that the cicada case study is an example of a genuinely explanatory application of mathematics to science" (Baker 2005, 235). Although he does not further substantiate this claim, I take it as starting point for a discussion of an extension of the D-N model to mathematical explanations.

At this point, the reader might be surprised to read that Baker suggests to rehabilitate, for a case of mathematical explanation, a model of scientific explanation that was addressed to explanations and predictions taking the form of logical derivations from observational statements. Our stupefaction, however, does not exclude that such a move might be fruitful, that it might reinforce previous criticisms or even have positive repercussions on the study of mathematical explanations. What is indisputable is that this move has not yet been made. Additionally, it should be noted that the standard problems with the D-N model do not obviously apply to the mathematical case. Indeed, many of the problems with the D-N model for scientific explanations arose from causality and probabilification, which are not so relevant to mathematical explanations (especially those within mathematics). And therefore the D-N model has prima facie a better chance of working with mathematical explanations.

In what sense is it possible for the D-N model to cover the cicada explanation? Baker remarks that the 5 steps explanation-schema above has a layout similar to the inferential layout proposed by the $\mathrm{D}-\mathrm{N}$ model. However, premise (2) ('prime periods minimize intersection') refers to a mathematical theorem, which does not have empirical content and does not represent a law of nature, thus violating conditions $R_{2}$ and $R_{3}$. Of course, Baker is conscious of this problem with the D-N requirements:

But does the deductive-nomological model have the resources to distinguish explanatory from non-explanatory components of the explanations? One point in the platonist's favour is that the purely mathematical premise (2) of the cicada inference is in the form of a general law, in this case a theorem of number theory. A broadening of the category of laws of nature to include mathematical theorems and principles, which share commonly cited features such as universality and necessity, would count the mathematical theorem (2) as explanatory on the same grounds as the biological law (1) (Baker 2005, 235).

In this passage he suggests that, in order to successfully cover the cicada explanation, the D-N model would need an extension based on the "broadening of the category of laws of nature to include mathematical theorems and principles". Although Baker does not push further this idea, if we follow his suggestion condition $\mathrm{R}_{2}$ of the original $\mathrm{D}-\mathrm{N}$ model would assume the following form:

$R_{2}{ }^{*}$ The explanans must contain general laws, which include mathematical theorems, and these must actually be required for the derivation of the explanandum.

Furthermore, also the logical condition of adequacy $\mathrm{R}_{3}$ should be modified in order for the model to admit mathematical explanations:

${ }^{9}$ Mathematical objects are acausal. Causal models miss MES simply because they consider explanation in the natural sciences as esssentially causal, and therefore they rule out the possibility of having mathematical explanations. 
$R_{3}{ }^{*}$ The explanans must have empirical or mathematical content.

What about conditions $\mathrm{R}_{1}$ and $\mathrm{R}_{4}$ ? When we come to the empirical condition of adequacy $\mathrm{R}_{4}$, it might be thought that this condition should be left out in the case in which the explanans contains mathematical statements. Observe, however, that it poses no problem once we adopt a view on truth-values of mathematical statements that appeals to the standards of mathematics itself. If we adopt such a point of view, known as mathematical naturalism (Maddy 1997), to defend the truth of a mathematical claim we do not need to appeal to standards outside of mathematics. Rather, we assume that mathematics provide their own suigeneris standards of justification, in the same way that physics does. On this account, the singular sentence 'Mt. Everest is snowcapped' is as true as the mathematical statement ' $7+5=12$ '. Of course, to preserve $\mathrm{R}_{4}$ with the name of 'empirical condition of adequacy' would be misleading in the context of our extension to mathematical explanations. This is why I will refer to this condition simply as $\mathrm{R}_{4}$ * ('The explanans must be true'). Finally, let's leave $\mathrm{R}_{1}$ unchanged and, for convenience sake, name it $\mathrm{R}_{1}$ * ('The explanandum must be a logical consequence of the explanans').

Let's baptize D-N*, or D-N Extended, the resulting account of explanation, whose criteria of explanation are fixed by conditions $\mathrm{R}_{1} * \ldots \mathrm{R}_{4} *$. On the $\mathrm{D}-\mathrm{N}^{*}$ account, a phenomenon or a mathematical fact is said to be explained when it is possible to deduce a statement (the explanandum $E^{*}$ ) from some statements (the explanans) according to the conditions of adequacy $\mathrm{R}_{1} * \ldots \mathrm{R}_{4} *$. Of course, with respect to the $\mathrm{D}-\mathrm{N}$ model, here the explanandum can be a statement describing an empirical phenomenon or a mathematical statement such as, for instance, 'For every real $x>0$ and every integer $n>0$ there is one and only one positive real $y$ such that $y^{n}=x$ '. The two situations correspond to a case of MES and MEM, respectively.

The important aspect of this extension is that, although some amendments have been introduced to cover mathematical explanations, the original and fundamental intuition behind the D-N model has been preserved: to have a genuine explanation is to have a sound deductive argument that makes use of at least one lawful connection. As specified by $\mathrm{R}_{2}$, the law-like generalizations considered here include mathematical theorems.

Finally, it might be noted that the advocates of the original D-N model referred to types of explanation where it is possible to trace a causal history from the sentences $C_{1}, C_{2}, \ldots, C_{k}$ to a singular event by empirical regularities $L_{1}, L_{2}, \ldots, L_{r}$ (Hempel and Oppenheim 1948, 139). Of course, to adopt conditions $\mathrm{R}_{1} * \ldots \mathrm{R}_{4} *$ is to abandon the idea that an explanation must provide such a causal linkage. Otherwise, no mathematical explanation would be allowed.

In this section I pursued further Alan Baker's suggestion and I showed how the D-N model could be modified to handle mathematical explanations inside its structure. This extension, which I called D-N*, has not been explored yet. To extend the basic intuition of the deductive-nomological model has required some changes to the criteria for sound explanation, however it has preserved the basic intuition of the original account proposed by Hempel and Oppenheim. This is why I consider the D-N* model as an extension of the preexistent D-N model, and not a new account of explanation. In the following section I shall offer two examples (of MES and MEM, respectively) that are 
intended to show that (and why) the D-N* account lacks resources to correctly account for mathematical explanations within its deductive structure. The reason for its failure will serve as a reinforcement of the dissatisfaction seen in the previous section.

\section{What's the Matter?}

It is now time to check the effectiveness of the $\mathrm{D}-\mathrm{N}^{*}$ model on cases which have been recognized as bona fide mathematical explanations. In this section I will consider two examples of mathematical explanation, one for MES and one for MEM in turn. What I am going to show is that a quick assessment of the $\mathrm{D}-\mathrm{N}^{*}$ model on these examples highlights two (related) difficulties that the Hempelian picture of explanation faces in the context of mathematical explanations in science and in mathematics: $(a)$ it cannot deal with mathematical operations or procedures that do not come under the form of statements but which are regarded as playing an explanatory role; $(b)$ it is not a sufficiently good indicator of the intuitions coming from scientific practice, thus imposing a picture of explanation that is not authentic. These two difficulties disclose two general problems: the D-N* model does not provide necessary conditions for mathematical explanation, i.e. there are cases which are regarded as bona fide mathematical explanations but which do not qualify as explanations according to the $\mathrm{D}-\mathrm{N}^{*}$ model; the $\mathrm{D}-\mathrm{N}^{*}$ model does not provide sufficient conditions for mathematical explanation, i.e. there are sets of statements that qualify as explanations according to the D-N model yet the scientists do not normally think of them as explanatory.

First of all, what about Baker's case of cicadas? Is the $\mathrm{D}-\mathrm{N}^{*}$ model able to recognize this case as a bona fide mathematical explanation? In cases of MES such as that of cicadas the explanandum, i.e. the sentence that describes the phenomenon to be explained, is a logical consequence of the explanans (as the 5-step argument above shows). Thus criterion $\mathrm{R}_{1} *$ is satisfied. In addition, it is straightforward to see that $\mathrm{R}_{2} *, \mathrm{R}_{3} *$ and $\mathrm{R}_{4} *$ are fulfilled too, thus making possible for the $\mathrm{D}-\mathrm{N}^{*}$ to consider the cicada example as a bona fide mathematical explanation. After all, we have built our extension of the original D-N model upon Baker's considerations concerning that particular case of MES. Nevertheless, not all MES exhibit the structure of the cicada explanation. There are MES that are recognized as such in scientific practice and in which the mathematical component of the explanation does not come in the form of a theorem, i.e. a mathematical statement (as required by $\mathrm{R}_{2}{ }^{*}$ ). For instance, in their paper "The Explanatory Power of Phase Spaces" (2008), Aidan Lyon and Mark Colyvan consider such an example of MES. They take into account a particular physical system called 'Hénon-Heiles system'. This system is formed by a particle moving in the bidimensional potential $U\left(q_{x}, q_{y}\right)=\frac{1}{2}\left(q_{x}^{2}+q_{y}^{2}\right)+q_{x} q_{y}^{2}-\frac{1}{2} q_{y}^{3}$. We want to explain the behaviour (regular or not) of the system for different energies ${ }^{10}$. Let's therefore take the sentence describing the (regular or chaotic) motion of the system as our explanandum. Now, it turns out that there are two mathematical routes to study the behaviour of the system. We can study the system through the Lagrangian analysis, or

\footnotetext{
${ }^{10}$ The problem of explaining the behaviour of this system was originally studied by the French astronomer Michel Hénon and his colleague Carl Heiles (Hénon and Heiles 1964). Lyon and Colyvan's study draws on their work.
} 
we can adopt the Hamiltonian formulation which comes with a particular mathematical structure called phase space. The Lagrangian formulation is obtained by introducing the Lagrangian function $L=T-U$, where $T$ is the kinetic energy of the system, and successively obtaining the equations of the motion from the so called Lagrange's equations. In this formulation, a system with $n$ degrees of freedom possesses $n$ (second-order) differential equations of motion, while the state of the system is represented by a point in an $n$-dimensional configuration space whose coordinates $q_{i}$ are named generalized coordinates. The Hamiltonian formulation, on the other hand, is "based on a fundamentally different picture" (Goldstein 1957, 335) and makes possible to describe the motion in terms of first-order equations of motion, known as Hamilton's canonical equations of motion. These equations describe the behaviour of the system point in a particular space, the phase space, which has $2 n$-dimensions and whose coordinates are the $2 n$ independent variables that appear in the canonical equations of motion. In other words, in the Hamiltonian formulation of mechanics the dynamics of our Hénon-Heiles system is defined by the evolution of points ('trajectories') in the phase space ${ }^{11}$.

The fact that the Hénon-Heiles system exhibits regular or chaotic motion is deduced visually from a representation in the phase space. How? By considering the total energy of the system $E$ constant, we lower the dimensionality of the phase space by one. Next we take a 2-dimensional cross section of this hypersurface in the phase space and we map the intersections of the trajectories with the plane by using a function called Poincaré map. Finally, we look at the dots made by the solutions (orbits of the system) on the Poincaré section and we can visually grasp qualitative information about the dynamics of the system at that particular energy. Thus the phase space, with its mathematical apparatus, is regarded to have an explanatory role:

The explanatory power is in the structure of the phase space and the Poincaré map. So it seems that this is a case where using the phase space is essential to our understanding and ability to explain certain features of the world. (Lyon and Colyvan 2008, 14)

Now, it is important to note again that the Hamiltonian procedure involving phase space is not the only alternative for the study of the system. However, to analyze the system via the Lagrangian route seems not to convey the sense of explanatoriness that we obtain from the use of the phase space theory in the Hamiltonian formalism:

[...] although there is a Lagrangian formulation of the theory in question that does not employ phase spaces, the cost of adopting such an approach is a loss of explanatory power. (Lyon and Colyvan 2008, 2)

From Lyon and Colyvan's example two important points emerge: first, even though mathematics comes as an essential ingredient, it is not a particular theorem (i.e. a mathematical law) which participates in the explanation; second, although two mathematical procedures are acceptable as to study the physical phenomenon (regular or chaotic motion of the particle moving in the potential), only one of them carries explanatory power. Consequently, in the context of this example, the $\mathrm{D}-\mathrm{N} *$ is confronted with the following problem: the model cannot deal with mathematical operations or procedures (such as

${ }^{11}$ Of course, there are technicalities I am glossing over here for the purposes of exposition. See Hénon and Heiles (1964) and Lyon and Colyvan (2008) for the full treatment. 
the use of the Hamiltonian formalism including phase space and Poincaré map) which do not come under the form of statements, and therefore it does not recognize the explanation as genuine ${ }^{12}$. What is more, even if we would have such mathematical procedures under the form of statements, the D-N* model would lack resources to discriminate between the explanatory mathematical procedure and the non-explanatory one. Indeed, it might be thought that what phase-space language provides is just a useful way of visualizing universal generalizations. Although the individuation of the general (mathematical) laws that are required for the derivation of the explanandum would be quite tedious, we might think that for some initial conditions we will have that such (mathematical) laws imply that the system has chaotic behaviour. According to this scenario, the D-N* would account for the explanatory character of the phase-space treatment. Nevertheless, in this case the Lagrangian treatment will be explanatory as well. After all, Hamilton's equations are derived from Lagrange's equations using a (mathematical) transformation, namely the Legendre transform. Consequently, also in the Lagrangian case it will be possible to say that some (mathematical) laws imply that, for certain initial conditions, the system has chaotic behaviour. More generally, these procedures (the Lagrangian and the Hamiltonian) are both formally correct from a mathematical point of view, and therefore the $\mathrm{D}-\mathrm{N}^{*}$ would consider both equally explanatory on the basis of criterion $\mathrm{R}_{1} *$ (both are good ingredients of the logical deduction). But, again, note that scientists consider as bona fide mathematical explanation only one mathematical procedure, that which uses the Hamiltonian formalism. As a consequence, to regard the Lagrangian treatment of the Hénon-Heiles system as equally explanatory, as the $\mathrm{D}-\mathrm{N}^{*}$ model suggests, would go against the opinion of scientists (Hénon and Heiles) and would not do justice to their intuitions.

In the previous lines I showed that the $\mathrm{D}-\mathrm{N}^{*}$ model does not recognize as explanatory the example of MES in question because of difficulty $(a)$. Furthermore, I claimed that even if the $\mathrm{D}-\mathrm{N}^{*}$ model would be capable of bypassing difficulty $(a)$, it would suggest a picture of explanation which does not fit with the intuitions coming from scientific practice. In other words, difficulty $(b)$ remains. The second example I want to consider is a case of mathematical explanation, and in particular a case of MEM, where the difficulty $(b)$ is even more pronounced. To anticipate the point, the $\mathrm{D}-\mathrm{N}^{*}$ model identifies every formal proof in mathematics as a genuine explanation. Every formal proof, in fact, inevitably follows a logical deductive schema in which the basic concept is that of a statement being a logical consequence of some other statements. Moreover, when we consider such a proof, conditions $\mathrm{R}_{2} *, \mathrm{R}_{3} *$ and $\mathrm{R}_{4} *$ are fulfilled as well. But, once again, does the $\mathrm{D}-\mathrm{N}^{*}$ model accurately mirror the intuitions coming from the practicing mathematicians? It seems that this is not the case, and this exactly because mathematicians do not consider every correct formal proof as an explanation of a mathematical result. There are several examples of MEM that have been discussed in the literature and that might be used here to illustrate this point (Mancosu 2011 provides a survey of various cases which are to the point). Nevertheless, for the purposes of this paper it will be sufficient to consider just one case.

${ }^{12}$ Note the difference between the Hénon-Heiles case and the example chosen by Baker, where the mathematics involved was expressed by a single theorem. 
In his paper "Explanatory Unification and the Causal Structure of the World" (1989), Philip Kitcher points out that explanatory asymmetries arise also in the domain of mathematics and are not a privilege of the causal debate on explanation (Kitcher 1989, 425) ${ }^{13}$. To illustrate his point he considers the proof of a property of finite groups by means of one specific axiomatization of the theory of finite groups. In that case, one particular axiomatization containing the existence of the inverse and idempotent elements is preferred by the mathematicians in order to explain why finite groups satisfy the division property. Nevertheless, the reverse derivation, i.e. the derivation of the existence of an idempotent element and of inverses from the division property, is regarded as a less natural and non-explanatory derivation (though formally valid). The former derivation is regarded as explanatory on the basis of its capacity of providing more general results, whereas the other derivation does not provide such possibility:

It is not hard to see a reason for the distinguishing of the derivations: the preferred derivation can be generalized to achieve more wide-ranging results [...] the explanatory derivation is similar to derivations we could provide for a more general result; the non-explanatory derivation cannot be generalized, it applies only to the local case (Kitcher 1989, 425)

Now, we want to analyze the situation through the lenses of the $\mathrm{D}-\mathrm{N}^{*}$ model. What we discover pretty soon is that, analogously to what happens when the D-N is confronted with cases of explanatory asymmetries in the empirical sciences, such as in the flagpole and the shadow example reported in section 2 , the D-N* model lacks resources to discriminate between the two (formally valid) proofs and pick out the bona fide mathematical explanation. Indeed, according to the $\mathrm{D}-\mathrm{N}^{*}$ model, both the mathematical deductions meet criteria $\mathrm{R}_{1} *-\mathrm{R}_{4}$, and are therefore recognized as genuine mathematical explanations. Certainly proving theorems is the canonical means of obtaining knowledge of mathematical facts. However, it seems that if we limit our picture of the cognitive activity informing mathematical reasoning to include just deductively valid arguments from indubitable premises, then we will not have any access to the sorts of reasons that we are interested in here. This is why the $\mathrm{D}-\mathrm{N}^{*}$ model, which is based on such a picture, does not discriminate between the explanatory derivation and the non-explanatory one in the example put forward by Kitcher.

It might be observed that the D-N model left room for a judgement of more/less explanatoriness on a continuum. In other words, that exist degrees of explanation. The basic idea is to subsume cases (i.e. explananda) under laws, so the more cases you subsume under the fewer and more basic laws, the more explanatory degree you get. Now, whether mathematicians recognise proofs as more and less explanatory, the same clue might be used to evaluate degrees of explanation in mathematical proofs through the D-N* model. Nevertheless, as in the case reported by Kitcher, mathematicians usually consider as the only option for a derivation to be explanatory/non-explanatory. Therefore it seems that this degrees-of-explanation idea would not save the D-N* model from the inadequacy highlighted so far.

The unconvinced reader might still think that the $\mathrm{D}-\mathrm{N}^{*}$ model can account for ex-

${ }^{13}$ This is the first time, at least to my knowledge, that such an analogy, i.e. the analogy between explanatory asymmetries in empirical science and explanatory asymmetries in mathematics, is brought to light. 
planatory proofs, and that D-N* explanations are sufficient in mathematics. After all, although only a few accounts of explanatory proofs in mathematics have been put forward, the prima facie impression may be that at least one of them seems to be rephrasable in terms of the D-N* schema. In his seminal 1978 paper on mathematical explanation in mathematics, Mark Steiner proposed his account for proof-explanations (Steiner 1978). According to Steiner, a proof is explanatory if: it depends on a property of a mathematical entity mentioned in the theorem (this property is called characterizing property); it can be deformed by substituting the characterizing property of a related mathematical entity and getting a related theorem. The general idea behind these criteria is that to every deformation of the proof there corresponds a deformation in the theorem, and therefore to an array of (explanatory) proofs there corresponds an array of theorems. In this case, the unconvinced reader might say that what Steiner's model picks out is a class of explanatory proofs that can be accounted in terms of an entire array of D-N* explanations. Observe, however, that Steiner's account has been strongly criticized by various authors (see Mancosu 2011 for a survey of these criticisms). More importantly, among these criticisms, some have pointed to the fact that Steiner's model does not correctly account for cases of mathematical explanations recognized as such in mathematical practice (Hafner and Mancosu 2005, Molinini 2012) ${ }^{14}$. Therefore it seems that difficulty (b) comes up again.

The general moral of the previous lines is that the $\mathrm{D}-\mathrm{N}^{*}$ model does not accurately mirror important aspects of scientific practice and, furthermore, it does impose a picture of explanation which is not authentic. By adopting the $\mathrm{D}-\mathrm{N}^{*}$ we are left with a schema which is nothing more than a purely logical deduction. Consequently, explaining an outcome $E$ (a statement describing an empirical phenomenon or a mathematical statement) is just a matter of showing that it is expectable on the basis of lawful connections (where laws also include mathematical theorems). Although this would be at odds with the indicators coming from scientific practice, as the two previous examples show, it might be thought that this observation cannot be used to criticize the D-N*. In fact, once we adopt the Hempelian perspective that explanation is logical deduction, we do not need to resort to such 'indicators' from scientific practice. It should be noted, however, that the import of such indicators is considered by many philosophers as of primary importance for the philosophical analysis of the notion of explanation in science and mathematics (Mancosu 2008). And, once again, it seems very natural to think that accounting for them is one of the main task of philosophy of science.

${ }^{14}$ In my (Molinini 2012) I show how, at least in some particular cases, the explanatory character of the proof depends on the purity of methods used by the mathematician. In considering a proof for a theorem, the mathematician restricts the conceptual resources used to prove the theorem to those which determine the content of the theorem. In this way he increases the epistemic quality of the proof, which he finally regards as explanatory. This intuition cannot be rendered through the D-N model, which does not account for such extra-logical ingredients. 


\section{Conclusions}

Despite the great interest in the linkage between scientific explanation and mathematical explanation, an extensive discussion of models of scientific explanation in the context of mathematical explanation has not been offered and work is just beginning. In this paper I showed how the D-N model of scientific explanation can be thought to extend to mathematical explanation when the conditions of adequacy on which it depends are properly modified. My analysis was intended to clarify the reasons why the picture of explanation which comes with the D-N model is insufficient to cover mathematical explanations. In fact, these reasons are not explicitly stated in the literature on explanation, although it is often assumed that the D-N model cannot cover mathematical explanations. Few authors (Alan Baker, Robert Batterman, Mauro Dorato and Laura Felline) have provided a sketch of the reasons why the D-N picture of explanation should be regarded as inadequate in the context of mathematical explanation. Nevertheless, rather than a complete analysis, these authors have proposed only some considerations in this direction. My analysis fills this gap because it is entirely focused on the applicability of the D-N picture of explanation to mathematical explanations, and in this sense it adds considerably to the lack of analysis present in much of the debate, while it goes beyond the (quick, again) considerations offered in the literature by Baker, Dorato and Felline, and Batterman.

It was not my intention here to discuss every possible way to extend the D-N model to mathematical explanation. I have picked out only one possible extension, which I named D-N*. By pointing to difficulties $(a)$ and $(b)$, I showed how the $\mathrm{D}-\mathrm{N}^{*}$ suffers problems of necessity and sufficiency: there exist mathematical explanations that don't fit the model but are regarded as bona fide explanations by the scientists; there are mathematical explanations that fit the model but that are not considered as genuine explanations in scientific practice. Moreover, I put forward a diagnosis of such difficulties. The failure of the $\mathrm{D}-\mathrm{N}^{*}$ model, as in the case of the original model, has do to with the inability of logic alone to mirror particular non-logical features of the case studies which carry explanatory import. In the two examples considered in the previous section these non-logical features are the capacity mathematics has of making possible to infer visually some information on a diagram and its capacity of providing more general results. Making logical deduction the hallmark of explanation preserves the basic intuition of the $\mathrm{D}-\mathrm{N}$ model but amounts to the imposition of a defining characteristic feature on what ought to be counted as 'mathematical explanation'. The resulting picture of explanation is incomplete and not satisfactory. And my general feeling is that every effort to extend the D-N picture of explanation to MES and MEM would face impediments similar to those highlighted here and would therefore be unsuccessful.

Of course, my dissatisfaction with the D-N and the D-N*, or better with the specific picture of explanation which stands behind these models, is based on the following assumption: the intuitions coming from scientific practice, although not providing normative and rigid standards of explanation, supply philosophers with some guidelines in the study of explanation. And these intuitions should be mirrored by our philosophical account(s) of explanation. Nevertheless, this is something which I regard as natural (and widely accepted) not only in the context of explanation, but more generally in the 
context of philosophy of science. I would be happy about the following situation: the D-N* model, or any other account of explanation, identifies a mathematical explanation as genuine and that particular explanation is recognized as a bona fide explanation in scientific practice as well. This situation would be, I think, an indicator of the fruitfulness of the philosophical investigation which lies behind that account of explanation. On the other hand, I regard neither reasonable nor philosophically fructuous that a philosophical model of explanation does impose on the scientist a normative criterion of explanation that the scientist would not accept.

As a whole, my results confirm the dissatisfaction that philosophers of science feel towards the use of the D-N model in the context of mathematical explanation. Furthermore, they highlight the reasons why the picture of explanation which stands behind that account is problematic, thus making our sense of dissatisfaction more satisfactory.

\section{REFERENCES}

Baker, A. 2005. Are there genuine mathematical explanations of physical phenomena? Mind 114: 223-238. Baker, A. 2009. Mathematical explanation in science. British Journal of Philosophy of Science 60: 611-633.

Batterman, R. 2002. The devil in the details. Oxford: Oxford University Press.

Batterman, R. 2005. Response to Belot's 'Whose devil? Which details?'. Philosophy of Science 72: 154-163.

Belot, G. 2005. Whose devil? Which details? Philosophy of Science 72: 128-153.

Bromberger, S. 1966. Why-questions. In Mind and Cosmos, edited by R. Colodny. Pittsburgh: University of Pittsburgh Press.

Dorato, M., and L. Felline. 2011. Scientific explanation and scientific structuralism. In Scientific Structuralism, edited by A. Bokulich and P. Bokulich, Volume 281 of Boston Studies in Philosopby of Science, 161-176. Dordrecht: Springer.

Goldstein, H. 1957. Classical Mechanics, 1st ed. Reading, Massachusetts: Addison Wesley.

Hafner, J., and P. Mancosu. 2005. The varieties of mathematical explanation. In Visualization, Explanation and Reasoning Styles in Mathematics, edited by P. Mancosu, K. F. Jørgensen, and S. Pedersen, 215-250. Dordrecht: Springer.

Hafner, J., and P. Mancosu. 2008. Beyond unification. In The Pbilosophy of Mathematical Practice, edited by P. Mancosu, 151-179. Oxford: Oxford University Press.

Hempel, C., and P. Oppenheim. 1948. Studies in the logic of explanation. Philosophy of Science Studies 15: 135-175.

Hénon, M., and C. Heiles. 1964. The applicability of the third integral of motion: Some numerical experiments. Astronomical Journal 69: 73-79.

Kitcher, P. 1989. Explanatory unification and the casual structure of the world. In Scientifical Explanation, edited by P. Kitcher and W. Salmon, Volume XIII of Minnesota Studies in the Philosophy of Science, 410-505. University of Minnesota Press, Minneapolis.

Lyon, A., and M. Colyvan. 2008. The explanatory power of phase spaces. Pbilosophia Mathematica 16: 227-243.

Maddy, P. 1997. Naturalism in mathematics. Oxford: Clarendon Press.

Mancosu, P, ed. 2008. The philosophy of mathematical practice. Oxford: Oxford University Press.

Mancosu, P. 2011. Explanation in mathematics. In The Stanford Encyclopedia of Philosophy (Summer 2011 ed.)., edited by E. N. Zalta.

Molinini, D. 2012. Learning from Euler. From mathematical practice to mathematical explanation. Philosophia Scientiae 16: 105-127. 
Saatsi, J. 2011. The enhanced indispensability argument: Representational versus explanatory role of mathematics in science. The British Journal for the Philosophy of Science 62: 143-154.

Salmon, W. 1971. Statistical explanation. In Statistical Explanation and Statistical Relevance, edited by W. Salmon, 29-87. Pittsburgh: University of Pittsburgh Press.

Salmon, W. 1989. Four decades of scientific explanation. In Scientifical Explanation, edited by P. Kitcher and W. Salmon, Volume XIII of Minnesota Studies in the Philosophy of Science. University of Minnesota Press, Minneapolis.

Sandborg, D. 1998. Mathematical explanation and the theory of why-questions. British Journal for the Philosophy of Science 49: 603-624.

Scriven, M. 1962. Explanation, prediction and laws. In Scientific Explanation, Space, and Time, edited by H. Feigl and G. Maxwell, Volume III of Minnesota Studies in the Philosophy of Science, 170-230. Minneapolis: University of Minnesota Press.

Steiner, M. 1978. Mathematical explanation. Pbilosophical Studies 34: 135-151.

Tappenden, J. 2005. Proof style and understanding in mathematics i: Visualization, unification and axiom choice. In Visualization, Explanation and Reasoning Styles in Mathematics, edited by P. Mancosu, K. F. Jørgensen, and S. Pedersen, 147-214. Dordrecht: Springer.

Daniele Molinini is a postdoctoral Fernand Braudel-IFER Fellow at the University of Barcelona (UB).

AdDRESS: LOGOS Group, Dept. Lògica, Història i Filosofia de la Ciència U. de Barcelona. C/ Montalegre, 6. Barcelona 08001 (Spain). E-mail: dmolinini@gmail.com 